Article

\title{
A Novel Method for Estimation of Femoral Neck Bone Mineral Density Using Forearm Images from Peripheral Cone Beam Computed Tomography
}

\author{
Kwanmoon Jeong ${ }^{1,2}$, Hoon Ko ${ }^{1,2}$, Chang-Hoon Lee ${ }^{2,3}$, Myeung Su Lee ${ }^{2,3}$, Kwon-Ha Yoon ${ }^{2,4, *}$ \\ and Jinseok Lee ${ }^{1,2, *}$ \\ 1 Department of Biomedical Engineering, Wonkwang University School of Medicine, 460 Iksandeaero, Iksan, \\ Jeonbuk 570-749, Korea; kor.kwanmoon@gmail.com (K.J.); idayfly8710@gmail.com (H.K.) \\ 2 Imaging Science based Lung and Bone Disease Research Center, Wonkwang University, 460 Iksandeaero, \\ Iksan, Jeonbuk 570-749, Korea; lch110@wonkwang.ac.kr (C.-H.L.); ckhlms@wku.ac.kr (M.S.L.) \\ 3 Department of Rheumatology Internal Medicine, Wonkwang University School of Medicine, \\ 460 Iksandeaero, Iksan, Jeonbuk 570-749, Korea \\ 4 Department of Radiology, Wonkwang University School of Medicine, 460 Iksandeaero, Iksan, \\ Jeonbuk 570-749, Korea \\ * Correspondence: khy1646@wku.ac.kr (K.-H.Y.); gonasago@wku.ac.kr (J.L.); \\ Tel.: +82-63-850-6970 (K.-H.Y. \& J.L.)
}

Academic Editor: Yang Kuang

Received: 29 February 2016; Accepted: 11 April 2016; Published: 19 April 2016

\begin{abstract}
The main goal of osteoporosis treatment is prevention of osteoporosis-induced bone fracture. Dual-energy X-ray absorptiometry (DXA) and quantitative computed tomographic imaging (QCT) are widely used for assessment of bone mineral density (BMD). However, they have limitations in patients with special conditions. This study evaluated a method for diagnosis of osteoporosis using peripheral cone beam computed tomography (CBCT) to estimate BMD. We investigated the correlation between the ratio of cortical and total bone areas of the forearm and femoral neck BMD. Based on the correlation, we established a linear transformation between the ratio and femoral neck BMD. We obtained forearm images using CBCT and femoral neck BMDs using dual-energy X-ray absorptiometry (DXA) for 23 subjects. We first calculated the ratio of the cortical to the total bone area in the forearm from the CBCT images, and investigated the relationship with the femoral neck BMDs obtained from DXA. Based on this relationship, we further investigated the optimal forearm region to provide the highest correlation coefficient. We used the optimized forearm region to establish a linear transformation of the form to estimate femoral neck BMD from the calculated ratio. We observed the correlation factor of $r=0.857$ (root mean square error $=0.056435 \mathrm{~g} / \mathrm{cm}^{2}$; mean absolute percentage error $=4.5105 \%$ ) between femoral neck BMD and the ratio of the cortical and total bone areas. The strongest correlation was observed for the average ratios of the mid-shaft regions of the ulna and radius. Our results suggest that femoral neck BMD can be estimated from forearm CBCT images and may be useful for screening osteoporosis, with patients in a convenient sitting position. We believe that peripheral CBCT image-based BMD estimation may have significant preventative value for early osteoporosis treatment and management.
\end{abstract}

Keywords: cone beam computed tomography (CBCT); cortical bone; forearm; femoral neck; correlation; linear transformation

\section{Introduction}

Osteoporosis is the most common amongst all metabolic bone disorders, characterized by low bone mass and micro-architectural deterioration of the bone tissue [1-5]. Dual-energy X-ray 
absorptiometry (DXA) and quantitative computed tomographic imaging (QCT) are widely used for the assessment of bone mineral density (BMD) and diagnosis of osteoporosis. DXA analysis is based on the principle that the bone areas decrease the signal of higher energy $X$-rays, while the soft tissue areas allow the signal to pass through. Thus, DXA images are analyzed to calculate bone threshold and identify bone boundary [6-9]. QCT is based on CT scanning with a calibration standard to convert Hounsfield Units (HU) to BMD values. These methods provide accurate BMD values and are clinically used for diagnosing osteoporosis [10-13]. However, these methods primarily scan the lumbar spine and hip, with patients in a supine position [12,14-16]; thus, these methods can only be applied to limited conditions such as the supine position. DXA and QCT-based scanning is difficult in patients who have undergone hip replacement. Furthermore, scanning of the lumbar spine and hip in a supine position may include genital organs, which may lead to uneasiness in the scanned patients. For these reasons, we investigated methods that could replace conventional BMD examination for diagnosis of osteoporosis, which can be performed more easily and with fewer limitations based on the fact that the cortical bone area is gradually reduced due to aging and is more severe for osteoporosis $[17,18]$. In this study, we observed a high correlation between the ratio of cortical and total bone areas in the forearm $\left(R^{c a / t a}\right)$ and femoral neck BMD. Our findings indicate that femoral neck BMD can be estimated using forearm images from peripheral cone beam computed tomography $(\mathrm{CBCT})$.

\section{Materials and Methods}

\subsection{Subjects and Measurements}

We obtained forearm images of 23 subjects using peripheral CBCT (PHION, Nano Focus Ray, Jeonju, Korea). The scanning parameters included $130 \mathrm{kvp}, 8 \mathrm{~mA}$, scanning times of $7.5 \mathrm{~s}$, and image matrixes of $1024 \times 1024$ pixels with slice thickness of $0.2162 \mathrm{~mm}$. The scanning regions were obtained roughly from the carpal bone to the elbow, which included the forearm midshaft. The total slice image number was 797. The study subjects consisted of 3 men and 20 women with a mean age of 63.3 years; among them, 14 and 9 were patients with osteoporosis patients and non-osteoporosis subjects, respectively. Their detailed demographic data are summarized in Table 1.

Table 1. Demographic data of the subjects.

\begin{tabular}{cccc}
\hline Subjects & $\begin{array}{c}\text { Non-Osteoporosis } \\
(\boldsymbol{n}=\mathbf{9})\end{array}$ & $\begin{array}{c}\text { Osteoporosis } \\
(\boldsymbol{n}=\mathbf{1 4})\end{array}$ & $\begin{array}{c}\text { All Subjects } \\
(\boldsymbol{n}=\mathbf{2 3})\end{array}$ \\
\hline Sex (n) & Male 2 & Male 1 & Male 3 \\
Age (years) & Female 7 & Female 13 & Female 20 \\
$50-59(\mathrm{n})$ & $63.3 \pm 9.57$ & $69.7 \pm 6.62$ & $67.2 \pm 8.32$ \\
$60-69(\mathrm{n})$ & 3 & 1 & 4 \\
$70-79(\mathrm{n})$ & 5 & 5 & 10 \\
Height $(\mathrm{cm})$ & $159.7 \pm 8.81$ & $151.9 \pm 8.34$ & $155 \pm 9.20$ \\
Weight $(\mathrm{kg})$ & $62.2 \pm 7.68$ & $51.2 \pm 8.80$ & $55.5 \pm 9.85$ \\
BMI $\left(\mathrm{kg} / \mathrm{m}^{2}\right)$ & $24.4 \pm 3.14$ & $22.1 \pm 2.19$ & $23.0 \pm 2.81$ \\
$\mathrm{BMD}^{\mathrm{f}}\left(\mathrm{g} / \mathrm{cm}^{2}\right)$ & $0.698 \pm 0.09$ & $0.5 \pm 0.07$ & $0.6 \pm 0.11$ \\
\hline
\end{tabular}

$\mathrm{BMD}^{\mathrm{f}}$ : femoral neck bone material density (BMD) obtained from X-ray absorptiometry (DXA). Values are means \pm standard deviation.

We used the forearm images to estimate femoral neck BMD, which were compared with reference femoral neck BMD values obtained by DXA (Discovery-W scanner, Hologic Inc., Bedford, MA, USA). The entire measurement procedure was performed by three radiologists in Wonkwang University Hospital (WKUH), who are inter-operators. The WKUH Institutional Review Board (IRB) approved the measurement and analysis protocol. 


\subsection{Computation of Forearm $R^{\text {calta }}$ from Peripheral Cone Beam Computed Tomography (CBCT) Images}

Using forearm images obtained by peripheral CBCT, we first calculated the $R^{\text {calta }}$. We extracted the areas from both cortical radius and ulnar bones as shown in Figure 1b. The cortical bone compartment was segmented by an auto-contouring process, which generates a periosteal contour that delineates mineralized bone and extra-osseal soft tissue, and an endosteal contour that delineates the endocortical boundary from the cancellous compartment $[19,20]$. To extract the periosteal surface, the thresholding which defined the cortical bone was applied to the CT image to obtain the contour of cortical bone. The endosteal surface was extracted by masking the periostea surface with bone mask from an original image, and the periosteal contour was peeled to leave only the marrow pixels [21]. Subsequently, we subtracted the endosteal surface from periosteal surface to obtain the cortical bone segmentation. The areas of cortical radius and ulna bones were calculated by counting the number of pixels inside the extracted cortical bone segment. Finally, the total radius and ulna bone areas were calculated by counting the total number of pixels of periosteal surface, and defined by $A r^{c}$ (radius) and $A r^{c}(u \ln a)$, respectively [22,23]. Next, we calculated the total radius and ulna bone areas by counting the total number of pixels within the cortical areas, as shown in Figure 1c. The total radius and ulna bone areas were defined by $A r^{t}$ (radius) and $A r^{t}$ (ulna), respectively. Each four-part area comprised of $A r^{c}$ (radius), $A r^{c}\left(\right.$ ulna), $A r^{t}$ (radius), and $A r^{t}(u l n a)$ was confirmed by two radiologists. Finally, we calculated $R^{c a / t a}$ in four different ways, as shown in Equations (1)-(4).

$$
\begin{aligned}
& R^{c a / t a}(\text { radius })=A r^{c}(\text { radius }) / A r^{t}(\text { radius }) \\
& R^{\text {ca/ta }}(u \ln a)=A r^{c}(u \ln a) / A r^{t}(u \ln a) \\
& R^{\text {ca/ta }}(\text { total })=\left[A r^{c}(\text { radius })+A r^{c}(\text { ulna })\right] /\left[A r^{t}(\text { radius })+A r^{t}(\text { ulna })\right] \\
& R^{\text {ca/ta }}(\text { average })=\left[R^{c a / t a}(\text { radius })+R^{c a / t a}(\text { ulna })\right] / 2
\end{aligned}
$$

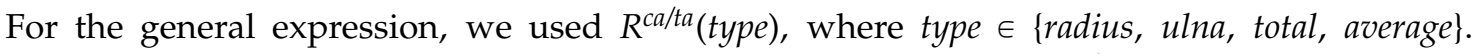
The four ratio types of the cortical bone area to the total bone area of $R^{\text {ca/ta }}($ type $)$ were repeatedly computed for all 797 slice images.

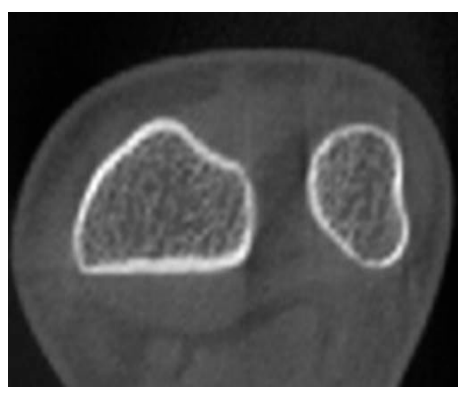

(a)

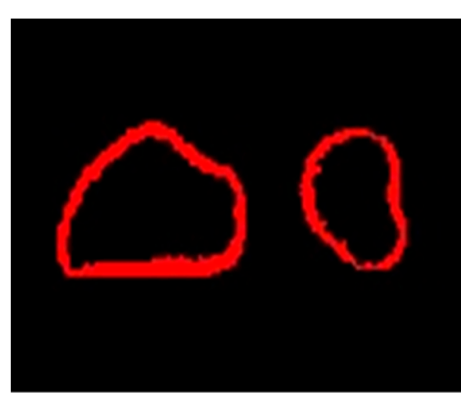

(b)

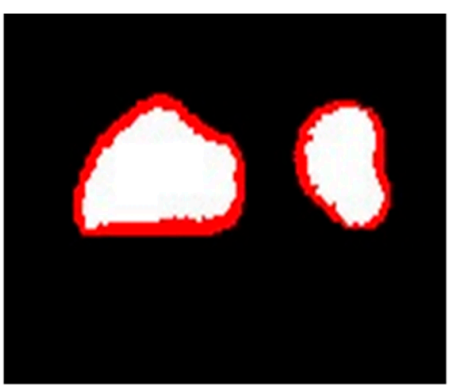

(c)

Figure 1. (a) Forearm images of the radius and ulna. (b) Separation of the cortical bones. (c) Total bone areas (red area + white area).

During the post-processing of all 797 slice images, the radiologists first identified the 50-slice midshaft area (region $M$ ) as shown in Figure 2. Note that 50 slices are equivalent to $10.8 \mathrm{~mm}$ in length (e.g., $50 \times 0.2162 \mathrm{~mm}$ ). Subsequently, the 50-slice image region in the proximal direction was defined as region $P 1$, and the other 50 slice image regions in the distal direction as $D 1$ to $D 8$. In this way, we classified into the 10 region with each 50 slice image region. More specifically, $R_{G}{ }^{c a / t a}($ type $)$ was defined as the mean value of $R^{\text {calta }}$ (type) in the region $G$, where $G \in\{D 1, D 2, D 3, D 4, D 5, D 6, D 7$, 
$D 8, M, P 1\}$. For instance, $R_{D 1}{ }^{c a / t a}$ (radius) represents the mean value of $R^{c a / t a}$ (radius) in the region $D 1$, while $R_{M}^{\text {ca/ta }}\left(\right.$ total) is the mean value of $R^{\text {calta }}($ total) in the region $M$.
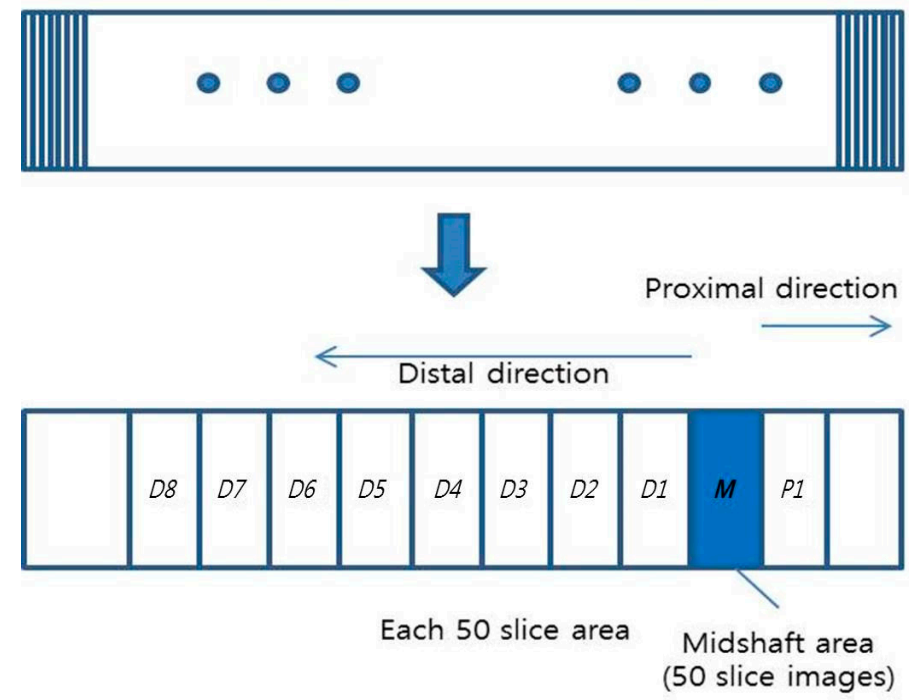

Figure 2. The obtained slice images were grouped into 10 regional areas, each with 50 slice images.

\subsection{Regression of Femoral Neck BMD from $R_{G}{ }^{\text {calta }}$ (type)}

We assumed that each $R_{G}{ }^{c a / t a}$ (type) and femoral neck BMD $\left(B M D^{f}\right)$ value was related by a linear transformation as described by Equation (5).

$$
B M D^{f} \approx e-B M D_{G}(\text { type })=k_{G}{ }^{1}(\text { type }) \cdot R_{G}^{\text {ca/ta }}(\text { type })+k_{G}{ }^{2}(\text { type })
$$

where $e-B M D_{G}($ type $)$ is the estimated BMD from $R_{G}{ }^{c a / t a}($ type $)$, and $k_{G}{ }^{1}($ type $)$ and $k_{G}{ }^{2}($ type $)$ are the scaling and offset constants, respectively. More specifically, Equation (5) can be re-expressed as

$$
\begin{gathered}
\left.\left.B M D^{f} \approx e-B M D_{G}(\text { radius })=k_{G}{ }^{1} \text { (radius) }\right) \cdot R_{G}{ }^{\text {ca/ta }}(\text { radius })+k_{G}{ }^{2} \text { (radius }\right) \\
B M D^{f} \approx e-B M D_{G}(\text { ulna })=k_{G}{ }^{1}(\text { ulna }) \cdot R_{G}{ }^{\text {ca/ta }}(\text { ulna })+k_{G}{ }^{2}(\text { ulna }) \\
B M D^{f} \approx e-B M D_{G}(\text { total })=k_{G}{ }^{1}(\text { total }) \cdot R_{G}{ }^{\text {calta }}(\text { total })+k_{G}{ }^{2}(\text { total }) \\
\left.\left.B M D^{f} \approx e-B M D_{G}(\text { average })=k_{G}{ }^{1} \text { (average }\right) \cdot R_{G}{ }^{\text {calta }}(\text { average })+k_{G}{ }^{2} \text { (average }\right)
\end{gathered}
$$

where the scaling and offset constants for $k_{G}{ }^{1}$ (type) and $k_{G}{ }^{2}$ (type) change according to each $R_{G}{ }^{\text {ca/ta }}$ (type). This assumption of linearity is supported by experimental data described in the Results section. The values of the constants $k_{G}{ }^{1}$ (type) and $k_{G}{ }^{2}$ (type) can be directly obtained by linear least squares regression. Furthermore, among Equations (6)-(9), we selected the equation that provided the overall minimum root mean square error (RMSE) and the highest correlation $r$ factor.

\subsection{Statistical Analyses}

Statistical analyses were performed using IBM SPSS Statistics for Windows, version 22.0 (IBM Corp., Armonk, NY, USA, 2012). The Pearson's correlation coefficients were used for calculating linear regression analysis. The correlation between $R_{G}{ }^{\text {calta }}($ type $)$ and $B M D^{f}$ was analyzed using correlation coefficient $r$. The linear regression lines with $k_{G}{ }^{1}($ type $)$ and $k_{G}{ }^{2}$ (type) were determined 
using the least-squares method. Root mean square error (RMSE) and mean absolute percentage error (MAPE) were calculated in order to evaluate this estimation as defined by

$$
\begin{gathered}
\text { RMSE }=\sqrt{\frac{1}{n} \sum_{i=1}^{n}\left(B M D^{f}{ }_{i}-e-B M D_{G}\left(\text { type }_{i}\right)^{2}\right.} \\
M A P E=\frac{1}{n} \sum_{i=1}^{n}\left|\frac{\left(B M D^{f}{ }_{i}-e-B M D_{G}\left(\text { type }_{i}\right)\right.}{B M D^{f}}\right| \times 100
\end{gathered}
$$

Given the established linear regression, the statistical $t$-test for regression slope was performed. The test determines whether there is a significant linear relationship between the independent variable $R_{G}{ }^{c a / t a}($ type $)$ and the dependent variable $B M D^{f}$. To apply the linear regression $t$-test, we calculated standard error of the slope and found the $p$-value. The $p$-value tests the null hypothesis that the slope is equal to zero (no effect). A low $p$-value $(<0.05)$ indicates that one can reject the null hypothesis. In other words, a predictor that has a low $p$-value is likely to be a meaningful addition to the regression model because changes in the predictor's value are related to changes in the response variable.

\section{Results}

\subsection{Correlation between $B M D^{f}$ and $R_{G}{ }^{c a / t a}$ (type)}

Figure 3 shows the correlation coefficient values between $B M D^{f}$ and $R_{G}{ }^{c a / t a}($ type), where type $\in\{$ radius, ulna, total, average $\}$ and $G \in\{D 1, D 2, D 3, D 4, D 5, D 6, D 7, D 8, M, P 1\}$. The correlation coefficient $r$ was highest in the $M$ and $D 1$ regions. The correlation coefficients tended to decrease in the regions further away from the region $M$ in both directions. Table 2 summarizes the correlation coefficient $r$ values in the $M$ and $D 1$ regions according to type. It shows that $B M D^{f}$ is the most correlated

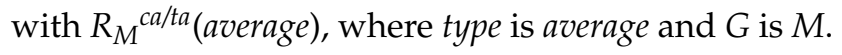

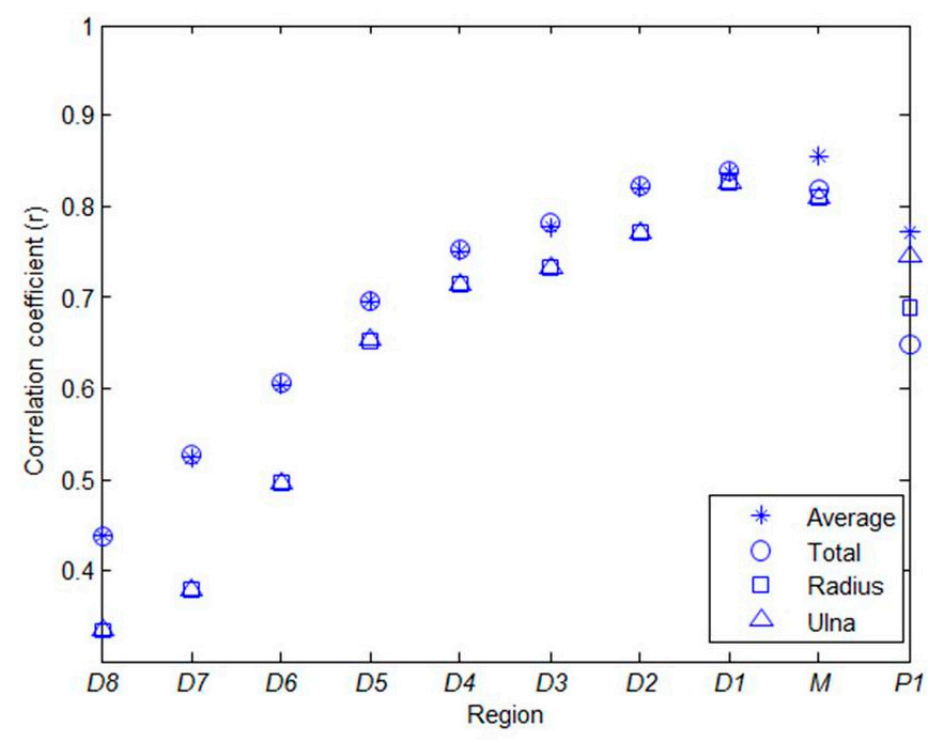

Figure 3. Correlation coefficient values between $B M D^{f}$ and $R_{G}{ }^{c a / t a}($ type), where type $\in$ \{radius, ulna, total, average $\}$ and $G \in\{D 1, D 2, D 3, D 4, D 5, D 6, D 7, D 8, M, P 1\}$. 
Table 2. Correlation coefficient $r$ values according to the type and the regions of $M$ and $D 1$.

\begin{tabular}{ccc}
\hline Correlation Coefficients & Region $\mathbf{D} \mathbf{1}$ & Region $\boldsymbol{M}$ \\
\hline$R^{c a / t a}($ radius $)$ & 0.823 & 0.815 \\
$R^{c a / t a}($ ulna $)$ & 0.785 & 0.807 \\
$R^{c a / t a}($ total $)$ & 0.835 & 0.827 \\
$R^{c a / t a}($ average $)$ & 0.834 & 0.857 \\
\hline
\end{tabular}

\subsection{Estimation of e-BMD ${ }_{G}$ (type) from $R_{G}{ }^{c a / t a}$ (type)}

Based on the observed high correlation coefficient $r$ values in the $D 1$ and $M$ regions, we investigated the linear transformation of the form in Equations (8) and (9), which correspond to total and average, respectively. For both total and average, we found the values of $k_{G}{ }^{1}($ type $)$ and $k_{G}{ }^{2}$ (type) with 1.3333 and -0.3333 , respectively, where $G$ includes $M$ and $D 1$.

We used the estimated $e-B M D_{G}\left(\right.$ type) to calculate the RMSEs and MAPEs from $B M D^{f}$. Table 3 summarizes the RMSEs and MAPEs between $e-B M D_{G}($ type $)$ and $B M D^{f}$, where type includes total and average, and $G$ includes $M$ and $D 1$. Similarly to the correlation coefficient $r$ values, e-BMD $D_{M}$ (average) provided the lowest values of RMSE and MAPE. The statistical $t$-test for regression slope also revealed there was a significant linear relationship between $e-B M D_{M}($ average $)$ and $B M D^{f}$.

Table 3. Root mean square errors (RMSEs) and mean absolute percentage errors (MAPEs) between e-BMD $D_{G}($ type $)$ and $B M D^{f}$.

\begin{tabular}{ccccc}
\hline Errors & RMSE (Root Mean Square Error) & MAPE (Mean Absolute Percentage Error) \\
\hline Region $(G)$ & $D 1$ & $M$ & $D 1$ & $M$ \\
$R_{G}{ }^{\text {calta }}($ total $)$ & 0.0609 & 0.0615 & 4.7091 & 4.9510 \\
$R_{G}{ }^{\text {calta }}($ average $)$ & 0.0609 & 0.0564 & 4.7175 & 4.5105 \\
\hline
\end{tabular}

\subsection{Analysis of $e-B M D_{G}($ type $)$}

Based on the results shown in Tables 2 and 3 we found that $e-B M D_{M}$ (average) provided both the highest correlation coefficient $r$ for $R_{M}^{\text {ca/ta }}$ (average) and the lowest RMSE and MAPE values. Figure 4 shows the Bland-Altman plot. A systematic bias of $-0.0043 \mathrm{~g} / \mathrm{cm}^{2}$ for $e-B M D_{M}$ (average) was detected, with a standard deviation of $0.05731 \mathrm{~g} / \mathrm{cm}^{2}$. The systematic bias in the $e-B M D_{M}$ (average) measurement can be corrected using a linear fit model. Figure 5 a shows the regression plot of $R_{M}{ }^{\text {calta }}$ (average) versus $B M D^{f}$, and Figure $5 \mathrm{~b}$ shows the regression plot of $e-B M D_{M}$ (average) versus $B M D^{f}$. In summary, the experimental relationship between $e-B M D_{M}$ (average) and $B M D^{f}$ was as follows:

$$
B M D^{f} \approx e-B M D_{M}(\text { average })=1.3333 \cdot R_{M}{ }^{\text {ca/ta }}(\text { average })-0.3333
$$




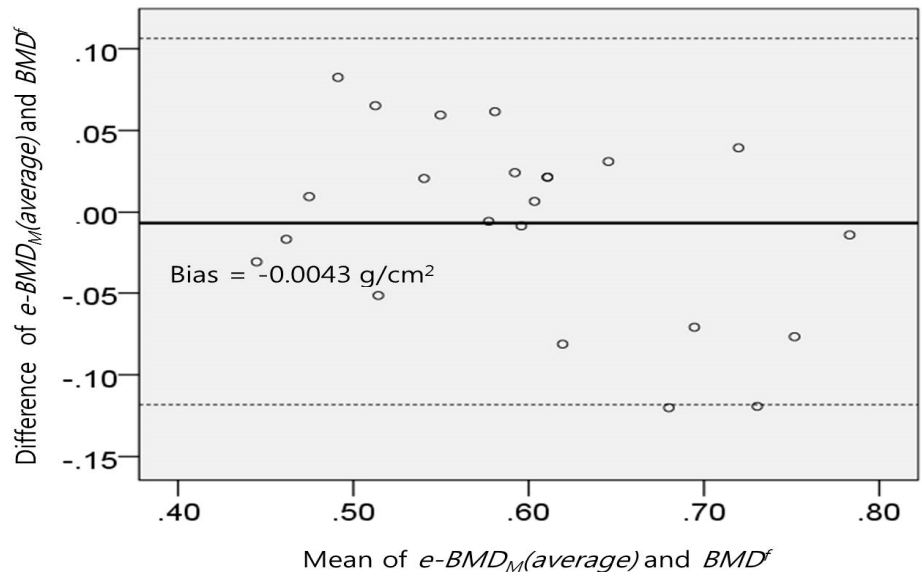

Figure 4. Bland-Altman plot: the mean of $e-B M D_{M}$ (average) and $B M D^{f}$ versus the difference of $e-B M D_{M}$ (average) and $B M D^{f}$. Area bone mineral density derived from dual-energy X-ray absorptiometry $\left(\mathrm{BMD}_{\mathrm{DXA}}\right)$ and computed tomographic images $\left(\mathrm{BMD}_{\mathrm{CBR}}\right)$. e-BMD $\mathrm{D}_{\mathrm{M}}$ (average) systematically underestimates $B M D^{f}$. The circle represents the difference of $e-B M D_{M}($ average $)$ and $B M D^{f}$ for each sample. Solid and dash lines represent mean $\pm 1.96 \times$ standard deviation of the differences of $e-B M D_{M}$ (average) and $B M D^{f}$.

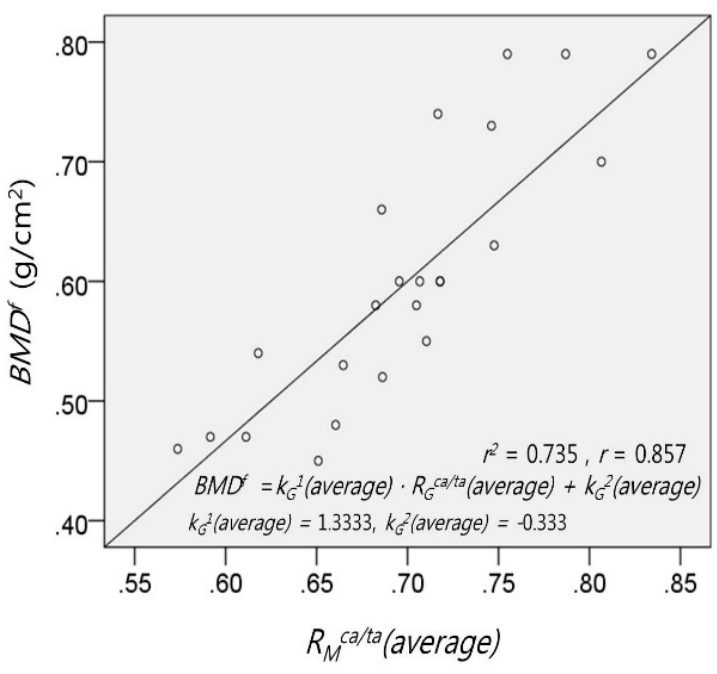

(a)

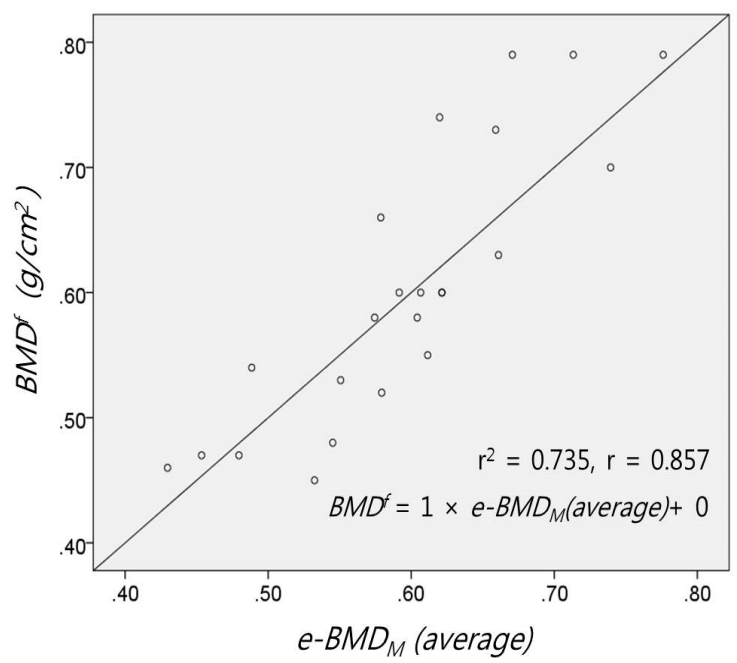

(b)

Figure 5. (a) Regression plot of $R_{M}{ }^{\text {ca/ta }}$ (average) versus $B M D^{f}$. (b) Correlation plot of $e-B_{M} D_{M}$ versus BMDf.

The correlation coefficient $r$ was $0.857\left(r^{2}=0.735\right)$. The RMSE and MAPE were $0.0564 \mathrm{~g} / \mathrm{cm}^{2}$ and $4.5105 \%$, respectively. These results show that there is a strong correlation between the $B M D^{f}$ from $e-B M D_{M}$ (average) and that it is possible to predict the $B M D^{f}$ on the basis of $e-B M D_{M}$ (average) from $R_{M}^{\text {calta }(\text { average). }}$

\section{Discussion}

Our proposed method is simple and easily reproducible because of the simple steps for BMD estimation. There are three main steps for the femoral neck BMD estimation. Firstly, the forearm bone CT images, especially for midshaft area, are obtained using CBCT. Secondly, cortical bone ratio of the forearm bone is measured automatically based on the cortices thickness to the amount of total bone. 
Lastly, the femoral neck BMD is estimated using the established equation between femur neck BMD and the measured ratio.

We further investigated the correlations of lumbar spine BMD $\left(B M D^{l s}\right)$ and total femur BMD $\left(B M D^{t f}\right)$ with $R_{M}{ }^{c a / t a}$ (Average), respectively. $B M D^{l s}$ and $B M D^{t f}$ were obtained in the same manner as for $B M D^{f}$ from DXA. Figure 6 shows the correlation of $R_{M}{ }^{\text {ca/ta }}$ (average) with $B M D^{l s}$ and $B M D^{t f}$, respectively. The correlation coefficients were $r=0.647$ and 0.792 , respectively, as summarized in Table 4 . Note that the correlation of $B M D^{f}$ corresponded to $r=0.857 . R_{M}{ }^{\text {ca/ta }}$ (average) was also correlated with BMDs of the lumbar spine and total femur. The higher correlation from femoral neck can be interpreted that the structure of femoral neck is similar with that of radius and ulna in forearm, which is cortical and trabecular bone structure.

Table 4. Correlation coefficient $r$ values of $R_{M}^{\text {ca/ta }}$ (average) with $B M D^{l s}$ and $B M D^{t f}$, respectively.

\begin{tabular}{cccc}
\hline Correlation Coefficients & $\boldsymbol{B M D}^{l s}$ & $\boldsymbol{B M D}^{t f}$ & $\boldsymbol{B M D}^{f}$ \\
\hline$R_{M}{ }^{\text {ca/ta }}($ average $)$ & 0.647 & 0.792 & 0.857 \\
\hline
\end{tabular}
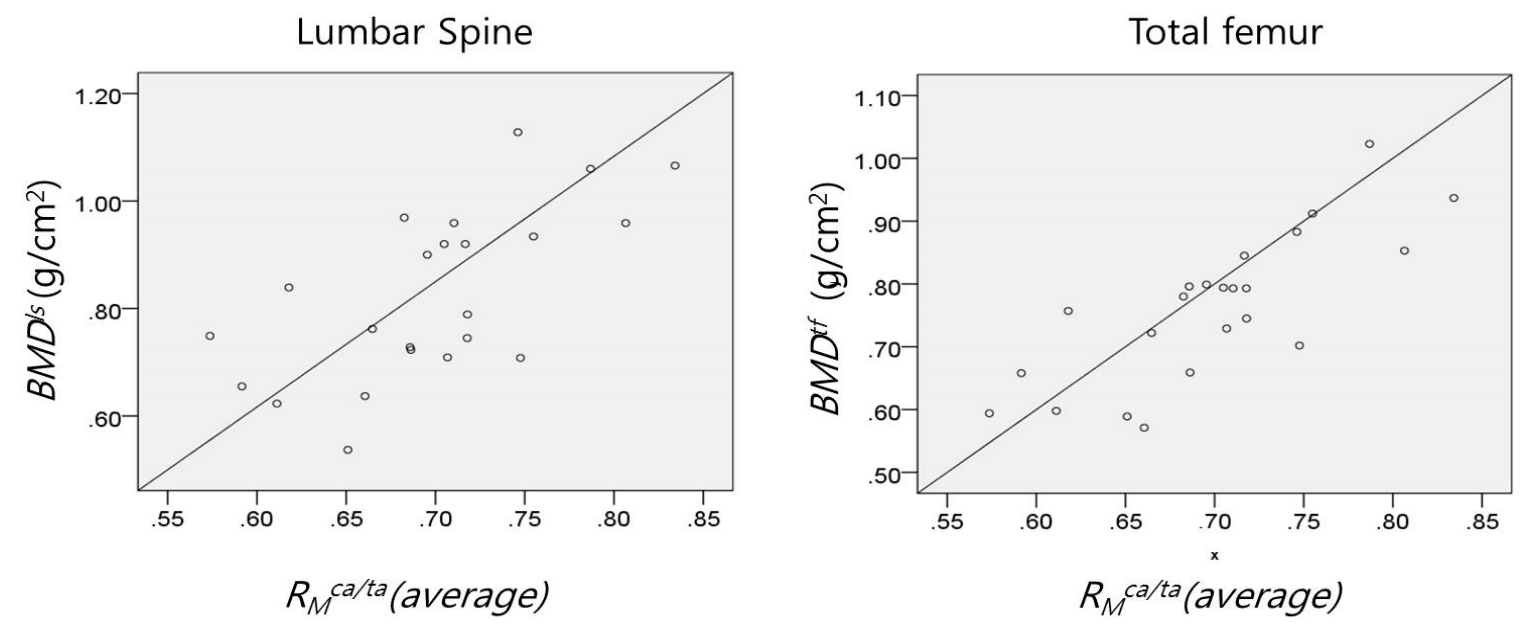

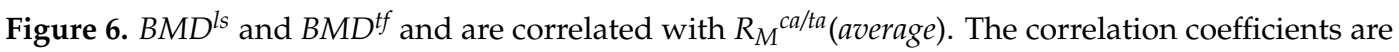
$r=0.647$ and $r=0.792$, respectively. Note that the correlation of $B M D^{f}$ corresponds to $r=0.857$.

Bone strength is determined by several factors of cortical thickness, bone size, trabecular bone density, and architecture. All these facts change with age as a result of the remodeling process. The cortical bone in a forearm and a femoral neck BMD also change with age $[17,18]$. However, there is a report that forearm BMD with DXA provides inadequate accuracy and very low predictive ability for identifying hip osteoporosis or femoral neck BMD [24]. Even though the femoral neck and the forearm bones are not directly associated with each other, similar research effort has been conducted on the relationship between BMDs from different sites. In [25-27], the results showed that that some parameters from the cortical bone in distal radius and ulnar on anteroposterior radiograph could reflect the femoral neck BMD. In [25], the distal radius BMD was correlated with the ratio of the outside cortical shell diameter to the inside cortical shell diameter at the metaphyseal/diaphyseal junction. In [26], radiologic density of dental structure from CBCT imaging could provide information of lumbar vertebra and femoral neck. In [27], the radial bicortical widths were measured at 50 and $70 \mathrm{~mm}$ proximal to the distal ulnar articular surface, and the mean bicortical width was compared with each patient's femoral BMD. The results showed that bicortical thickness of the distal radius was positively correlated with femoral neck BMD. It reflects similarity in quality and loading properties of the femur and radius as appendicular bones. 
In our study, we targeted the entire range of a forearm, and found the best position providing the highest correlation value. In addition, since the absolute value of bone size or volume may affect the BMD estimation, we considered the ratio value of cortical and total bone areas of the forearm. For further analysis, we investigated the correlation values between femoral neck BMD and other 12 parameters: volumes and areas in different areas: cortical volume in radius, total volume in radius, cortical volume in ulna, total volume in radius, average cortical volume in radius and ulna, average total volume in radius and ulna, cortical area in radius, total area in radius, cortical area in ulna, total area in ulna, average cortical area in radius and ulna, and average total area in radius and ulna, where the area is the midshaft. Figure 7 summarizes the comparison of the correlation coefficient values according to the volumes and area in different sites. It shows that the correlation coefficients from the volumes and areas in different areas were lower than the values from our proposed method.

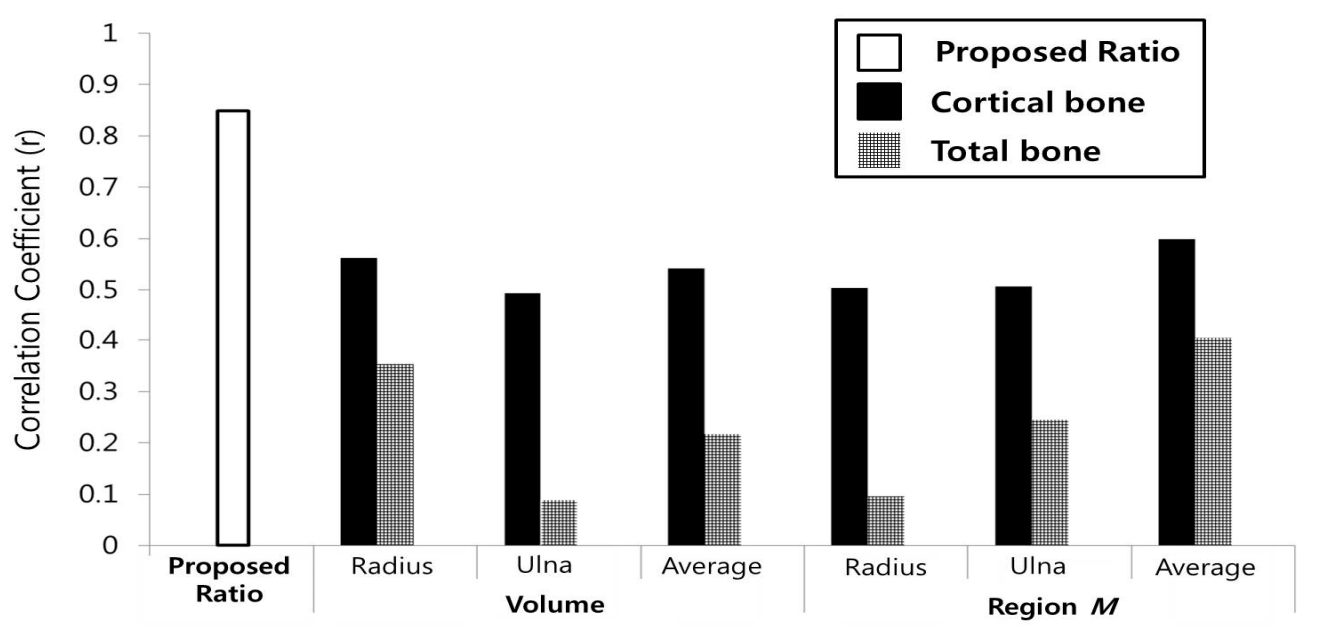

Figure 7. Comparison of correlation coefficient values according to volumes and areas in different sites.

In addition, one of the advantages in our method is that a forearm, especially the midshaft area, needs to be exposed via X-ray. On the other hand, using DXA and QCT, the entire axial skeleton near femur neck is exposed via $X$-ray. Because the cancer occurrence by $X$-ray exposure is one of the main factors, the $X$-ray exposure of the forearm midshaft area is better than that of the axial skeleton near femur neck. Obviously, we expect the deviated results between two groups having a job requiring upper body labor and a job requiring lower body labor. For the future research, the job-matching-based research needs to performed for elaborating the relationship.

Furthermore, this pilot study $(N=23)$ needs to be rigorously further validated by considering more extensive subjects. We further validated our proposed method with five healthy subjects with age of $27.6 \pm 2.71$. We calculated the ratio of the cortical to the total bone area in the forearm and estimated the femoral BMD based. The RMSE and MAPE were $0.028 \mathrm{~g} / \mathrm{cm}^{2}$ and $2.86 \%$, respectively. Even though the validation subject number is small, we found the additional data were fitted in our proposed method. We hope more related studies are performed in the near future.

The CBCT technique was recently introduced two decades ago and currently widely used for clinical diagnosis, especially for peripheral and dental use [28-31]. It provides two-dimensional and three-dimensional images for the radiographed area with a relatively low cost as compared to conventional CT. The radiation dose is comparable to that of panoramic imaging in the case of small field of views (FOVs). Due to the low cost and low radiation dose, it became popular, its use widespread. However, the gray values from CBCT are considered approximate values, which are not accurately expressed as HU comparing with conventional CT. There is a report that the gray values obtained by $\mathrm{CBCT}$ are higher than the $\mathrm{HU}$ values obtained by conventional $\mathrm{CT}$ of the same region [32]. The inaccuracy of gray values by CBCT may result in the incorrect boundary of cortical bone. As the density becomes weaker, the boundary extraction for the cortical bone becomes inaccurate. 
In our experiments, the more severe the osteoporosis was, the lower the accuracy for the boundary extraction was. For the case of the inaccurate boundary extraction, three radiologists in Wonkwang University Hospital confirmed and manually corrected the boundary when possible. Nevertheless, unlike most other studies, we utilized our own simple algorithm for the BMD estimation without any additional programs or calibration phantoms that are neither available nor easy to use by most researchers. In order to increase the accuracy of the femoral neck BMD, more research effort should be directed to the radiographic density for the boundary extraction in severe osteoporosis patients at the clinical level.

\section{Conclusions}

We observed a high correlation between the ratio of the cortical bone area to the total bone area in the forearm obtained from CBCT images and femoral neck BMD obtained from DXA images. Our results suggest that DXA-based femoral neck BMD can be accurately estimated using forearm $\mathrm{CBCT}$ images and may be used in an osteoporosis screening system, with patients placed in a convenient sitting position. Peripheral CBCT-based BMD measurement may provide significant value for prevention and early treatment and management of osteoporosis.

Acknowledgments: This study was partially supported by a grant from the Korean Health Technology R\&D Project, Ministry of Health and Welfare, Republic of Korea (HI12C0110), and the Korea Evaluation Institute of Industrial Technology (KEIT) (grant No. 10047759) funded by the Ministry of Trade, industry \& Energy (MOTIE).

Author Contributions: Kwanmoon Jeong and Hoon Ko collected, analyzed, and interpreted image data and drafted the initial manuscript. Chang-Hoon Lee and Myeung Su Lee performed DXA and CBCT scanning under IRB approval, and confirmed the image analysis findings. Kwon-Ha Yoon confirmed and validated the overall algorithm from the clinical perspective, and participated in manuscript writing and revision. Jinseok Lee conceived the main idea and participated in writing the final manuscript. All authors read and approved the final manuscript.

Conflicts of Interest: The authors declare no conflict of interest.

\section{References}

1. Kanis, J.A. Diagnosis of osteoporosis and assessment of fracture risk. Lancet 2002, 359, 1929-1936. [CrossRef]

2. Schuit, S.; van der Klift, M.; Weel, A.; de Laet, C.; Burger, H.; Seeman, E.; Hofman, A.; Uitterlinden, A.; van Leeuwen, J.; Pols, H. Fracture incidence and association with bone mineral density in elderly men and women: The rotterdam study. Bone 2004, 34, 195-202. [CrossRef] [PubMed]

3. Guglielmi, G.; Muscarella, S.; Bazzocchi, A. Integrated imaging approach to osteoporosis: State-of-the-art review and update. Radiographics 2011, 31, 1343-1364. [CrossRef] [PubMed]

4. Aitken, J. Relevance of osteoporosis in women with fracture of the femoral neck. BMJ 1984, 288, 597-601. [CrossRef] [PubMed]

5. Christodoulou, C.; Cooper, C. What is osteoporosis? Postgrad. Med. J. 2003, 79, 133-138. [CrossRef] [PubMed]

6. Yang, J.Y.; Kim, Y.M. Correlation analysis of BMD in proximal femur and spine. J. Korean Soc. Fract. 2003, 16, 570-576.

7. Blake, G.M.; Fogelman, I. Bone densitometry and the diagnosis of osteoporosis. Semin. Nucl. Med. 2001, 31, 69-81. [CrossRef] [PubMed]

8. Jang, J.; Yang, S.; Kim, D.; Jo, Y.; Lee, S.; Kim, K. Comparative study of femur BMD and lumbar BMD measurement using dual energy $\mathrm{X}$-ray absorptiometry in proximal femur fractures. Korean J. Bone Metab. 1996, 3, 170-173.

9. Ralston, S.H. Bone densitometry and bone biopsy. Best Pract. Res. Clin. Rheumatol. 2005, 19, 487-501. [CrossRef] [PubMed]

10. Engelke, K.; Libanati, C.; Liu, Y.; Wang, H.; Austin, M.; Fuerst, T.; Stampa, B.; Timm, W.; Genant, H.K. Quantitative computed tomography (QCT) of the forearm using general purpose spiral whole-body CT scanners: Accuracy, precision and comparison with dual-energy X-ray absorptiometry (DXA). Bone 2009, 45, 110-118. [CrossRef] [PubMed] 
11. Black, D.M.; Bouxsein, M.L.; Marshall, L.M.; Cummings, S.R.; Lang, T.F.; Cauley, J.A.; Ensrud, K.E.; Nielson, C.M.; Orwoll, E.S. Proximal femoral structure and the prediction of hip fracture in men: A large prospective study using QCT. J. Bone Miner. Res. 2008, 23, 1326-1333. [CrossRef] [PubMed]

12. Lang, T.; Guglielmi, G.; van Kuijk, C.; de Serio, A.; Cammisa, M.; Genant, H. Measurement of bone mineral density at the spine and proximal femur by volumetric quantitative computed tomography and dual-energy X-ray absorptiometry in elderly women with and without vertebral fractures. Bone 2002, 30, 247-250. [CrossRef]

13. Guglielmi, G.; Schneider, P.; Lang, T.; Giannatempo, G.; Cammisa, M.; Genant, H. Quantitative computed tomography at the axial and peripheral skeleton. Eur. Radiol. 1997, 7. [CrossRef]

14. Mochizuki, N.; Sugino, N.; Ninomiya, T.; Yoshinari, N.; Udagawa, N.; Taguchi, A. Association of cortical shape of the mandible on panoramic radiographs with mandibular trabecular bone structure in japanese adults: A cone-beam CT-image analysis. Oral Radiol. 2014, 30, 160-167. [CrossRef]

15. Engelke, K.; Adams, J.E.; Armbrecht, G.; Augat, P.; Bogado, C.E.; Bouxsein, M.L.; Felsenberg, D.; Ito, M.; Prevrhal, S.; Hans, D.B. Clinical use of quantitative computed tomography and peripheral quantitative computed tomography in the management of osteoporosis in adults: The 2007 ISCD official positions. J. Clin. Densitom. 2008, 11, 123-162. [CrossRef] [PubMed]

16. Vijay, A.; Shankar, N.; Ligesh, C.; Anburajan, M. Evaluation of osteoporosis using CT image of proximal femur compared with dual energy X-ray absorptiometry (DXA) as the standard. In Proceedings of the 2011 3rd International Conference on Electronics Computer Technology (ICECT), Kanyakumari, India, 8-10 April 2011; pp. 334-338.

17. Mosekilde, L. Age-related changes in bone mass, structure, and strength-Effects of loading. Z. Rheumatol. 2000, 59 (Suppl. S1), 1-9. [CrossRef] [PubMed]

18. Laval-Jeantet, A.-M.; Bergot, C.; Carroll, R.; Garcia-Schaefer, F. Cortical bone senescence and mineral bone density of the humerus. Calcif. Tissue Int. 1983, 35, 268-272. [CrossRef] [PubMed]

19. Ma, W.-Y.; Manjunath, B.S. Edgeflow: A technique for boundary detection and image segmentation. IEEE Trans. Image Process. 2000, 9, 1375-1388. [PubMed]

20. Shah, S.; Abaza, A.; Ross, A.; Ammar, H. Automatic tooth segmentation using active contour without edges. In Proceedings of the 2006 Biometrics Symposium: Special Session on Research at the Biometric Consortium Conference, Baltimore, MD, USA, 19 September-21 August 2006; pp. 1-6.

21. DeFreitas, S.C. Computer Method for Filling a Selected Region in a Target Image. U.S. Patent 5870100, 9 February 1999.

22. Burghardt, A.J.; Buie, H.R.; Laib, A.; Majumdar, S.; Boyd, S.K. Reproducibility of direct quantitative measures of cortical bone microarchitecture of the distal radius and tibia by HR-PQCT. Bone 2010, 47, 519-528. [CrossRef] [PubMed]

23. Buie, H.R.; Campbell, G.M.; Klinck, R.J.; MacNeil, J.A.; Boyd, S.K. Automatic segmentation of cortical and trabecular compartments based on a dual threshold technique for in vivo micro-CT bone analysis. Bone 2007, 41, 505-515. [CrossRef] [PubMed]

24. Trivitayaratana, W.; Trivitayaratana, P. The accuracy of bone mineral density at distal radius on non-forearm osteoporosis identification. J. Med. Assoc. Thail. 2001, 84, 566-571.

25. Rausch, S.; Klos, K.; Gras, F.; Skulev, H.K.; Popp, A.; Hofmann, G.O.; Mückley, T. Utility of the cortical thickness of the distal radius as a predictor of distal-radius bone density. Arch. Trauma Res. 2013, 2. [CrossRef] [PubMed]

26. Barngkgei, I.; Al Haffar, I.; Khattab, R. Osteoporosis prediction from the mandible using cone-beam computed tomography. Imaging Sci. Dent. 2014, 44, 263-271. [CrossRef] [PubMed]

27. Webber, T.; Patel, S.P.; Pensak, M.; Fajolu, O.; Rozental, T.D.; Wolf, J.M. Correlation between distal radial cortical thickness and bone mineral density. J. Hand Surg. 2015, 40, 493-499. [CrossRef] [PubMed]

28. Chen, B.; Ning, R. Cone-beam volume CT mammographic imaging: Feasibility study. Med. Imaging 2001, 4320, 655-664.

29. Scarfe, W.C.; Farman, A.G. What is cone-beam CT and how does it work? Dent. Clin. N. Am. 2008, 52, 707-730. [CrossRef] [PubMed]

30. Casselman, J.; Gieraerts, K.; Volders, D.; Delanote, J.; Mermuys, K.; de Foer, B.; Swennen, G. Cone beam CT: Non-dental applications. JBR-BTR 2013, 96, 333-353. [CrossRef] [PubMed] 
31. Cho, P.S.; Johnson, R.H.; Griffin, T.W. Cone-beam CT for radiotherapy applications. Phys. Med. Biol. 1995, 40. [CrossRef]

32. Arisan, V.; Karabuda, Z.C.; Avsever, H.; Özdemir, T. Conventional multi-slice computed tomography (CT) and cone-beam CT (CBCT) for computer-assisted implant placement. Part I: Relationship of radiographic gray density and implant stability. Clin. Implant Dent. Relat. Res. 2013, 15, 893-906. [CrossRef] [PubMed]

(c) 2016 by the authors; licensee MDPI, Basel, Switzerland. This article is an open access article distributed under the terms and conditions of the Creative Commons Attribution (CC-BY) license (http://creativecommons.org/licenses/by/4.0/). 